\title{
ANNULAR PANCREAS CAUSING DUODENAL OBSTRUCTION: A CASE REPORT
}

\author{
Swish Kumar Singh', Dinesh Kumar Agrawal2, Suganita 3 , G. N. Singh4 , Amar Kumar Singh ${ }^{5}$ \\ ${ }_{1}^{1}$ Post Graduate, Department of Radiodiagnosis, Patna Medical College \& Hospital, Patna. \\ 2 Post Graduate, Department of Radiodiagnosis, Patna Medical College \& Hospital, Patna. \\ ${ }^{3}$ Post Graduate, Department of Dermatology, Leprology \& Venereology, Patna Medical College \& Hospital, Patna. \\ ${ }^{4}$ Professor and HOD, Department of Radiodiagnosis, Patna Medical College \& Hospital, Patna. \\ ${ }_{5}^{5}$ Assistant Professor, Department of Radiodiagnosis, Patna Medical College \& Hospital, Patna.
}

\begin{abstract}
Annular pancreas is a rare congenital anomaly characterized by the band of pancreatic tissue of variable width partially or completely encircling the duodenum. This abnormality, although at times clinically silent or may be the cause of a broad spectrum of diseases. Complications range from neonatal intestinal obstruction to more complex pathologies in the adult such as pancreatitis, duodenal stenosis or duodenal or gastric ulceration. This condition is important to recognise, because radiologists are usually the first person to diagnose such condition. We report the case of a young patient of 10 years old female hospitalized for epigastric pain and repeated episodes of vomiting, in whom radiological investigations showed an annular pancreas. No other congenital anomaly of the intra-abdominal organs was noted. Both the rarity of this congenital abnormality and its probability of successful correction by surgical means have prompted us to make the following presentation.
\end{abstract}

\section{KEYWORDS}

Annular Pancreas, Duodenum, MRCP, ERCP.

HOW TO CITE THIS ARTICLE: Singh SK, Agrawal DK, Suganita, et al. Annular pancreas causing duodenal obstruction: a case report. J Evolution Med Dent Sci 2016;5(5):296-300, DOI: 10.14260/jemds/2016/64

\section{INTRODUCTION}

Annular pancreas is a rare congenital morphological anomaly of the pancreatic ducts.[1] It is formed due to failure of normal migration of the ventral pancreatic bud, which results in a part of pancreatic tissue encircling the duodenum. It is one of the few medical conditions, which can present itself in a wide range of clinical severities. It usually affects neonates, but in the elderly it can mimic a wide range of clinical entities like peptic ulcer, pancreatitis, obstructive jaundice, etc., thereby making the diagnosis difficult. At present imaging modalities as Computerized Tomography (CT), Endoscopic Retrograde Cholangiopancreatography (ERCP) and Magnetic Resonance Cholangiopancreatography (MRCP) are used to arrive at a diagnosis. Each modality though has its own limitations. Annular pancreas is diagnosed with nearly equal frequency in children and adults. The factors initiating symptoms are, duodenal obstruction, recurrent pancreatitis and duodenal or gastric ulceration. One new case of duodenal obstruction due to annular pancreas is presented.

\section{CONSENT}

Written informed consent was obtained from the patient for publication of this case report and accompanying images.

\section{CASE PRESENTATION}

A 10-year-old female presented with a few years history of pain upper abdomen, nausea and recurrent episodes of vomiting. The symptoms had become more frequent in the last few months.

Financial or Other, Competing Interest: None.

Submission 16-12-2015, Peer Review 17-12-2015,

Acceptance 30-12-2015, Published 18-01-2016.

Corresponding Author:

Dr. Swish Kumar Singh,

C/o., Paras Nath Prasad Singh,

71/B, Shivalik Apartment,

New Patliputra Colony,

Patna-800013, Bihar.

E-mail: swish_pmch@yahoo.com

DOI:10.14260/jemds/2016/64
The pain was present on and off. It was burning in nature, mild to moderate in intensity, poorly localized in the upper abdomen and was relieved by vomiting. Vomiting was recurrent, bilious and non-projectile. The episodes of vomiting used to occur post-prandially. There was no history of hematemesis. On examination the patient was of thin built. General physical examination was essentially normal. His vital parameters were within normal limits. Abdominal examination did not reveal any mass or any organomegaly and did not contribute significantly.

Routine biochemical and hematological investigations were within normal limits. Barium meal of the patient revealed dilated first part of the duodenum and narrowed second part of the duodenum with some extrinsic compression (Fig. 1). Ultrasonography of the abdomen was showing pancreatic head encircling the bowel loop through which nasogastric tube was passing (Fig. 2). An abdominal CT revealed a ring of pancreatic tissue encircling the second part of duodenum with no significant lymphadenopathy or any mass lesion. (Fig. 3). Axial and coronal T2W images show the same with narrowed $2^{\text {nd }}$ part of duodenum (Fig. 4).

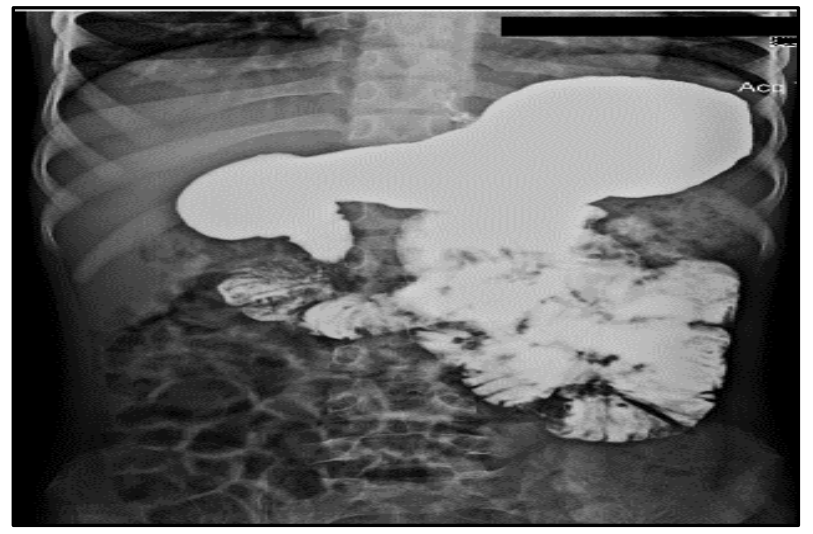

Fig. 1: Upper gastrointestinal barium study of the pyloroduodenal area revealing as concentric duodenal narrowing at the post bulbar area 


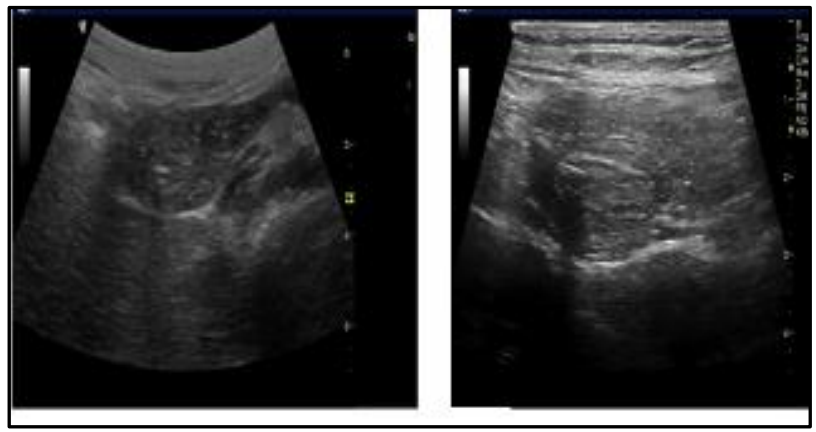

Fig. 2: USG revealing head of pancreas encircling the bowel loop with nasogastric tube in situ

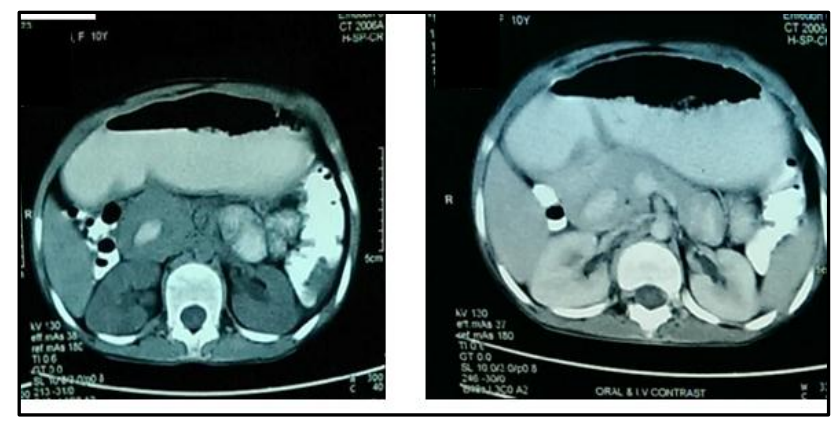

Fig. 3: CT of abdomen showing the annular pancreas encircling the second part of the duodenum. Central region of high attenuation representing contrast material within the narrowed duodenum

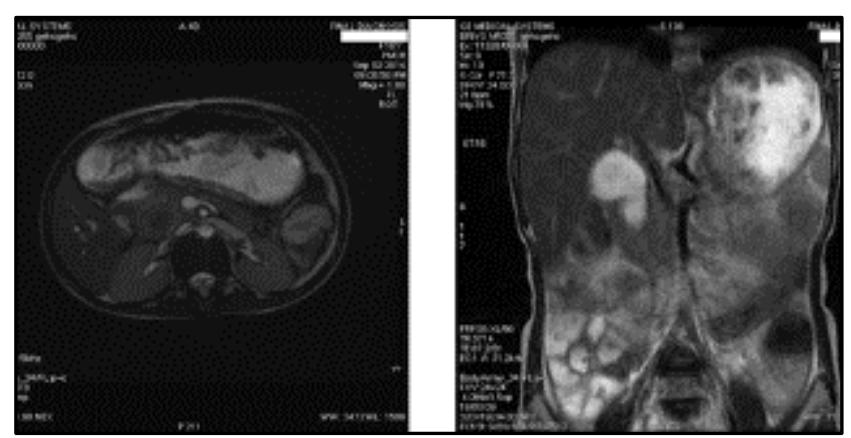

Fig. 4: Axial and Coronal MRI showing normal-appearing pancreatic tissue encircling the second part of duodenum

\section{DISCUSSION}

There are three types of pancreatic fusion anomalies, pancreas divisum, annular pancreas and portal annular pancreas with the first being commonest and latter being the rarest of all of them. [2] Annular pancreas is an embryological defect of the foregut. The pancreas develops from a dorsal and two ventral buds that first appear in the fifth gestational week as outgrowths of the primitive foregut. By the seventh gestational week, expansion of the duodenum causes the ventral bud to rotate and pass behind the duodenum from right to left and fuse with the dorsal bud.

The ventral bud forms the posterior head and uncinate process, whereas the dorsal bud forms the anterior head, body, and tail. Following this fusion, the ductal systems anastomose, a complicated process with a wide spectrum of possible outcomes. The portion of the ventral duct between the dorsalventral fusion and major papilla is termed the duct of Wirsung. The portion of the dorsal duct upstream to the dorsal-ventral fusion point is called the main pancreatic duct.
The segment of the dorsal duct downstream to the dorsal-ventral fusion point is termed the duct of Santorini or accessory pancreatic duct, which drains at the papilla minor.

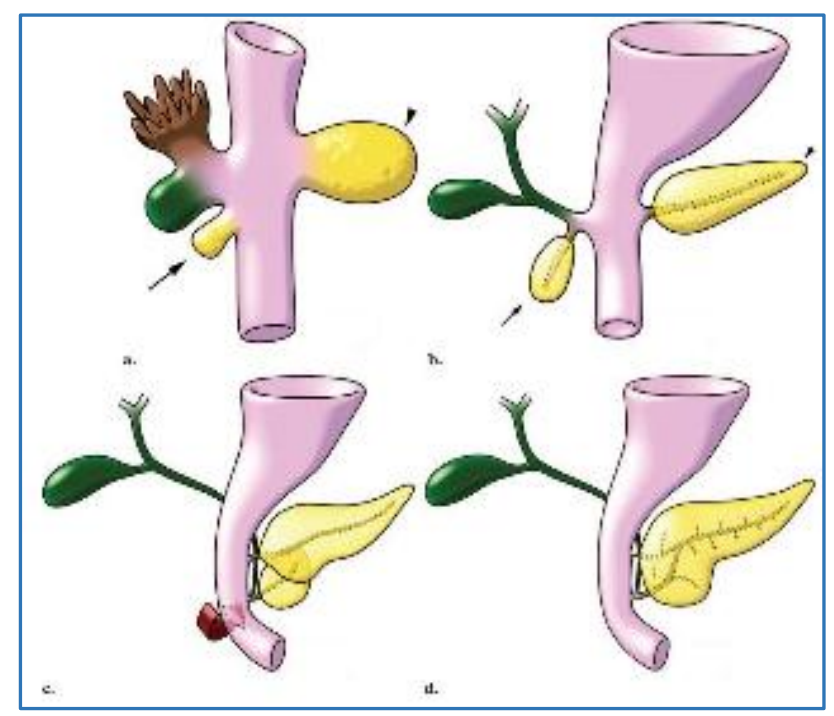

Fig. 5

Drawings illustrate the normal embryologic development of the pancreas and biliary tree. The ventral pancreatic bud (Arrow in a and b) and biliary system arise from the hepatic diverticulum and the dorsal pancreatic bud (Arrowhead in $\mathrm{a}$ and $\mathrm{b}$ ) arises from the dorsal mesogastrium. After clockwise rotation of the ventral bud around the caudal part of the foregut, there is fusion of the dorsal pancreas (Located anterior) and ventral pancreas (Located posterior). Finally, the ventral and dorsal pancreatic ducts fuse, and the pancreas is predominantly drained through the ventral duct, which joins the Common Bile Duct (CBD) at the level of the major papilla. The dorsal duct empties at the level of the minor papilla.

\section{Normal Anatomy \\ Pancreas}

The pancreas is a coarsely lobulated gland typically $15-20 \mathrm{~cm}$ in length and located in the anterior pararenal space of the retroperitoneum. The gland can be divided into four parts: head, neck, body, and tail. The pancreatic head is located within the curve of the duodenum to the right of the superior mesenteric vein. The uncinate process is a prolongation of the caudal part of the head, which is oriented toward the left. It has a triangular appearance and its anteromedial border can be straight or concave. The pancreatic neck is the constricted portion to the left of the head and ventral to the superior mesenteric vein. The pancreatic body and tail are located behind the lesser sac and the stomach. The border between the body and tail is not clearly defined but can be determined using one-half of the distance between the neck and the end of the pancreas.

The diameter of the MPD is normally $3.5 \mathrm{~mm}$ in the head, $2.5 \mathrm{~mm}$ in the body and $1.5 \mathrm{~mm}$ in the tail and the length of the MPD varies from 9.5 to $25 \mathrm{~cm}$. There are approximately 27 possible ductal configurations. Typically, the MPD has 20-30 side branches that enter the duct at right angles. The duct of Wirsung unites with the CBD and drains into the major papilla. The duct of Santorini, or accessory duct, drains the anterior and superior portion of the head into the minor papilla. 
The distal CBD and duct of Wirsung traverse the sphincter of Oddi (Which consists of three separate smooth muscles) to enter the duodenum. In most cases (80\%-90\%), the CBD and duct of Wirsung unite within this sphincteric segment with the muscular wrap being $10-15 \mathrm{~mm}$ in length.

\section{Classification}

Annular pancreas can be either complete or incomplete.

\section{Complete Annular Pancreas}

Pancreatic parenchyma or annular duct is seen to completely surround the $2^{\text {nd }}$ part of duodenum.

\section{Incomplete Annular Pancreas}

Annulus does not surround the duodenum completely, giving a 'Crocodile jaw' appearance.

Several theories have been proposed to explain the development of annular pancreas. Leeco's and Baldwin's theories are the most acceptable among all. Leeco postulated that the tip of the ventral bud fuses abnormally to the duodenal wall and rotates incorrectly around the duodenum and resulting in a band of fibrous or pancreatic parenchymal tissue around the second part of the duodenum.[3,4] Baldwin in 1910 reported that this condition arose because of the abnormal movement of the ventral pancreatic bud.[5]

The third theory explained by Verga in 1972, suggests that the primary abnormality is duodenal with the pancreas "filling the space" around a narrowed duodenum. This results in a complete or incomplete stenosis of the duodenal lumen. An alternative theory posits that hypertrophy of the dorsal and ventral buds results in a complete band of pancreatic tissue around the duodenum.[6] (A complete ring is found in approximately $25 \%$ of the cases).

It is a rare congenital anomaly (The incidence is approximately $1-3$ in 20000) and it is the most common anomaly of the pancreatic ducts after pancreas divisum. It was first reported by Tieddmann.[7] in 1818 and named as annular pancreas by Ecker.[8] in 1862. The first surgical treatment for an obstructive annular pancreas was performed by Vidal in 1905.[9] The rarity of the condition was supported by Vasconcelos and Sadek who reported a single case in 22,243 autopsies.[10] However, this number may not indicate the true prevalence because duodenal dissection is not routinely performed during autopsy. An incidence of $1.14 \%$ has been reported by Karasaki et al. based on institutional review of CT scans. However, D2 is involved in $74 \%$ of the cases.

Only 737 cases have been reported in the English literature till date with a slight female preponderance. Majority of the reported cases have been reported in the infant/new-born population. With the improvements achieved in imaging techniques, the diagnosis of AP increased in frequency. Yogi et al. classified AP in six types depending on site of drainage of annular duct.[11] The most common variety is type I, in which annular duct flows directly into the main pancreatic duct. In Type II, Wirsung duct encircles the duodenum, but still drains at the major papilla. The other four types are much less common.

It has been estimated that only about $33 \%$ of the cases are symptomatic. The nature of presentation differs according to patient age, although most presentation occurs most commonly in infancy or early childhood. Complications arising due to the presence of an annular pancreas are a rare cause of neonatal or childhood presentation. In infants/new-borns, annular pancreas appears most often in the first weeks of life by symptoms related to duodenal obstruction. In this scenario, it may often be present with various other congenital anomalies.[12] Symptoms may appear within the first few hours of life. Signs of neonatal intestinal obstruction may initially be nonspecific including poor feeding, vomiting and irritability. If proximal to the ampulla of Vater, vomiting may be non-bilious and therefore could be confused with less severe, non-life-threatening conditions. Children frequently present with Gastrointestinal (GI) symptoms including poor feeding, vomiting and abdominal distention. In few geographical areas most patients present late with malnutrition, failure to thrive, bile-stained vomiting and less frequently abdominal cramps.

The spectrum of clinical presentation in adult population is however quite variable. The age of revelation is usually between 20 and 50 years. The most frequently found symptoms are: upper abdominal pain (70\%), vomiting and nausea $(47 \%)$ and they are generally reflecting a proximal intestinal obstruction.[13] Other clinical manifestations have been reported: peptic ulcer secondary to stasis upstream of duodenal stenosis, acute, chronic or recurrent pancreatitis.[14] due to the default flow of pancreatic secretions in the annular pancreas and rarely jaundice due to the common bile duct stenosis by the annular pancreas or related to a lithiasic origin. It may rarely be associated with malignancy.

Clinical examination findings will vary according to the age at presentation and the extent of systemic upset. When presenting as intestinal obstruction, the abdomen will be distended (Before decompression) and there may be palpable peristalsis. Bowel sounds are variable. If presentation is delayed, there may be clinical signs of hypovolemic shock including pallor, poor capillary refill and drowsiness or lethargy.

There is a strong association between annular pancreas and other congenital abnormalities - up to $71 \%$ of cases have coexisting congenital anomalies. The most common association is with Down syndrome (Trisomy 21). However, there may be a wide range of associated cardiac (Heart defects) and gastrointestinal anomalies (Including Hirschsprung's disease and imperforate anus) as well as tracheo-oesophageal fistula, oesophageal atresia, common mesentery and Intraductal Papillary Mucinous Tumour (IPMT).

\section{INVESTIGATIONS \\ Prenatal Diagnosis}

It is becoming increasingly possible to detect the presence of annular pancreas in foetal life (2nd trimester). A nonspecific diagnosis of duodenal obstruction can be made prenatally with identification of simultaneous dilatation of the stomach and duodenum - the "double bubble" sign. This does not predict the specific cause of the duodenal obstruction and certainly is not specific to the diagnosis of annular pancreas. Indeed, presence of an annular pancreas is the cause in only $1 \%$ of cases of neonatal duodenal obstruction. Therefore, there is a search for more specific prenatal markers of annular pancreas. Several groups have shown that the presence of hyperechogenic bands around the duodenum specifically indicates the presence of an annular pancreas. 
This finding can now be used to specifically detect the presence of annular pancreas during foetal life.

\section{IMAGING MODALITIES \\ Abdominal Radiography}

The plain abdominal radiograph will not provide diagnostic structural information about the pancreas, in part due to the lack of tissue contrast. Cross-sectional imaging modalities are much more useful in delineating the precise anatomy of this area. However, if obstruction is complete, duodenal obstruction classically appears as a double bubble on abdominal radiograph. This represents dilatation of the stomach (Seen in the left upper quadrant) and proximal duodenum (Seen in the right upper quadrant). A double bubble on abdominal radiograph is sufficient to diagnose a complete duodenal obstruction. However, an upper gastrointestinal contrast radiograph is helpful for diagnosing a partial duodenal obstruction.

\section{Ultrasonography}

The use of ultrasonography (US) allows a real-time evaluation of the paediatric pancreas without the use of ionising radiation and usually without the need for sedation. This modality provides a safe, relatively cheap, and often readily available technique for imaging the paediatric pancreas. The reduced body wall thickness of the child in combination with the anatomical "window" provided by the liver, ensures that a better quality of pancreatic image is obtained in the child, as compared to the adult.

\section{Computed Tomography}

Recent improvement in spiral Computed Tomography (CT) technology, such as multidetector and subsecond rotation time, have made it possible to scan and pancreas with a collimation of $1 \mathrm{~mm}$ and less. The superior resolution in the zaxis and multiplanar reconstruction images allows the selection of the optimal sectional planes for the assessment of pancreas. The lack of intra-abdominal fat in children, combined with the often thin band of pancreatic tissue around the duodenum, may make identification of the annular pancreas with Computed Tomography (CT) difficult.

Despite this, detection rates can be greatly improved with a specific, Intravenous (IV)-contrast enhanced, pancreatic study. The use of CECT in adults is limited by the narrowness of the ring and by the fact that the pancreatic ring may lie intramurally in the duodenum without any plane existing in between. CT clearly delineates the ventral pancreatic ring encircling the duodenum. (A complete ring is not required for diagnosis).In fact, $75 \%$ of annular pancreas cases have a partial ring around the duodenum CT may also highlight extrinsic compression of the duodenum, which must be differentiated from thickening of the wall of the duodenum. CT may be used for further investigation of abnormalities detected with ultrasound imaging.

\section{Magnetic Resonance Imaging}

Magnetic Resonance Imaging (MRI) is an alternative to CT imaging for evaluation of the pancreas. Magnetic Resonance Cholangiopancreatography (MRCP) is being used with increasing frequency in the non-invasive evaluation of the biliary tree and pancreatic duct. It may depict the course and drainage pattern of the pancreatic duct and easily diagnose developmental anomalies of the pancreas. MRCP is especially appropriate for the assessment of congenital pancreatic anomalies, since it depicts ductal anatomy rapidly and noninvasively without risk of acute pancreatitis.

Even in this era of radiological sophistication, diagnosis may require surgical confirmation in more than $40 \%$ of the cases as each imaging modality has its own limitations. The diagnosis of annular pancreas used to be based on duodenography, which showed a medio-duodenal stenosis corresponding to the pancreatic ring. The Endoscopic Ultrasound (EUS) also allows approaching the diagnosis by showing the ring of normal pancreatic tissue encircling the duodenum, it can assess not only the pancreatic duct but also the parenchyma. ${ }^{[15]}$ The pancreato MRI (MRCP) remains the most reliable diagnostic tool and allows to highlight the presence of a pancreatic duct encircling the duodenum; however, requires a dilated ductal system.

Endoscopic Retrograde Cholangiopancreatography (ERCP) can also make the diagnosis, but it is invasive and can precipitate/exacerbate pancreatitis, besides the presence of a narrowed lumen may preclude its use. Laparotomy may be required to determine the cause of obstruction if this is not specifically identified on preoperative imaging. Tapering of the bowel is not necessary, even if the proximal segment is grossly dilated.

A diagnosis of annular pancreas should be kept in mind even in the adult population. Tuberculosis may be an important differential diagnosis in endemic countries. Treatment options need to be individualized.

\section{Management}

In clinically silent cases of annular pancreas, no specific intervention is required. Specific management will depend upon the nature of the complication or symptoms caused by the annular pancreas.

\section{Duodenal Obstruction}

Initially, management must include decompression with a nasogastric tube and fluid resuscitation. It is appropriate for patients to be transferred to a special care/high dependency unit, if available where appropriate monitoring and interventions can be instigated. Delayed presentation may result in significant systemic upset and it is vital that adequate resuscitation be carried out before surgery. Prior to surgery, fluid and electrolyte resuscitation is an absolute requirement. Transanastomotic nasogastric tubes may be necessary in centers where parenteral nutrition is not available, due to the delay in enteral feeding as a result of the dilated proximal segment.

Ultimately surgical intervention is required in cases of duodenal obstruction, even if the precise cause of the obstruction is unclear. Its aim is relief of the duodenal obstruction. In those who present as duodenal obstruction a simple enteric diversion procedure is done with duodenoduodenostomy being the preferred procedure. It has the advantage of being the most physiological by having the least blind loop segment. Gastrojejunostomy (With or without gastric resection) requires an additional vagotomy, especially in the young because of the propensity to cause ulcerations of the stoma of gastrojejunostomy.[16] Inflammations and adhesions in the $\mathrm{C}$ loop may preclude the possibility of a duodenojejunostomy. 
Although reported in literature, pancreatic resection is associated with a higher incidence of complications as pancreatitis, pancreatic fistulae and pancreatic insufficiency. Even the minor degrees of pancreatic leaks that go unnoticed on a gross level can cause an intense local inflammatory response, which can induce an intense local inflammatory and fibrotic reaction causing variable degree of luminal compromise of surrounding structures. Pancreatic resection is reserved to those rarest cases where malignancy cannot be excluded. Furthermore, the division of the annular pancreas is often followed by persistent symptoms, particularly abdominal pain (Up to $50 \%$ of cases).

\section{Prognosis and Outcomes}

Successful treatment of pediatric intestinal obstruction requires rapid identification, resuscitation and definitive management. In addition to the technical skill required to bypass the obstruction caused by aberrant pancreatic tissue, appropriate perioperative care is required to ensure a successful outcome after surgery. Surgical treatment of annular pancreas has an excellent prognosis as long as there is sufficient appropriate perioperative and postoperative care. This includes appropriately trained anaesthetic staff, ventilatory support and the facility for parenteral nutrition. Ultimately and despite appropriate surgical intervention to relieve duodenal obstruction if present the outcome may be affected by the presence of severe associated congenital abnormalities.

\section{Prevention}

With the improvement in prenatal diagnosis-in particular the identification of specific markers for annular pancreas detection of this congenital abnormality can be optimized. Although it cannot be prevented, earlier identification of complications in particular, intestinal obstruction will help to ensure that appropriate treatment can be instigated as soon as possible. Urgent surgical intervention is required in cases of duodenal obstruction caused by annular pancreas. Surgery is not required to correct annular pancreas if there are no complications caused by its presence. Prognosis after surgery for duodenal obstruction is good; however, overall outcome will also be affected by the severity of associated congenital anomalies.

\section{CONCLUSION}

Annular pancreas is a rare congenital malformation that manifests itself primarily by signs related to duodenal stenosis. The diagnosis is currently based on abdominal CT scan and MRI of the pancreas. Treatment is exclusively surgical, and a by-passing procedure is the method of choice in the treatment of annular pancreas producing duodenal obstruction. Both the rarity of this congenital abnormality and its successful correction by surgical means have prompted us to make this presentation.

\section{REFERENCES}

1. Kiernan PD, ReMine SG, Kiernan PC. Annular pancreas. Mayo clinic experience from 1957 to 1976 with review of literature. Arch Surg 1980.

2. Nijs E, Callahan M, Taylor G. Disorders of the pediatric pancreas: imaging features. Pediatr Radiol 2005;35:358373.

3. Dowsett JF, Rode J and Russell RCG (1989). Annular pancreas: a clinical, endoscopic and immunohistochemical study. Gut, 30, 130-135.

4. Leeco TM. Zur Morphologie des Pancrease. Sitzungsb Akad Wiss Math-Nat Ki Wien 1910;119:391-406.

5. Baldwin WM. A specimen of annular pancreas. Anat Rec 1910;4:299-304.

6. Glazer G, Margulis A. Use of ERCP in Annular pancreas. Radiology 1979;133:303-306.

7. Tieddmann F. Uber die Verschiedenheiten des Ausfuhrungsgangs der Bauchpeicheldruse bei dem Menshen und Sauugetiern. Dtsch Arch Physiol 1818;4:403.

8. Ecker A (1862). Bildungsfehier des Pankreas und des Herzens. Z Art Med, 14, 354.

9. Vidal E (1905). 18e Congress de Chirugie, Paris, 1905. Proces Verbaux, Memoires et discussion (Assosc Franc Chiru), 18, 739.

10. Vasconcelos E, Sadek HM. Pancreas annular produzindoestenose duodenal. Rev Bras Gastroenterol 1949;1:535-551.

11. Yogi $Y$, Shibue $T$ and Hashimoto S (1987). Annular pancreas detected in adults, diagnosed by endoscopic retrograde cholangiopancreatography: Report of four cases. Gastroenterologia Japonica, 22, 92-99.

12. Zyromski N, Sandoval J, Pitt H, et al. Annular pancreas: dramatic differences between children and adults. J Am Coll Surg 2008;206:1019-1025.

13. Urayama S, Kozarek R, Ball T. Presentation and treatment of annular pancreas in an adult population. Am J Gastroenterol 1993;88:467-468.

14. Ohno Y, Kanematsu T. Annular pancreas causing localized recurrent pancreatitis in a child: report of a case. Surg Today 2008;38:1052-1055.

15. Kandpal H, Bhatia V, Garg P, et al. (2009). Annular pancreas in an adult patient: diagnosis with endoscopic ultrasonography and magnetic resonance cholangiopancreatography. Singapore Medical Journal, 50, e29.

16. Thomford NR, Knight PR, Pace WG, et al. (1972). Annular pancreas in the adult: Selection of operation. Annals of Surgery, 1972, 159-162. 